\title{
Measurement of fracture energy of concrete at high strain rates
}

\author{
V. Rey-de-Pedraza ${ }^{1, *}, F$. Gálvez ${ }^{1}$, and D. Cendón Franco ${ }^{1}$ \\ ${ }^{1}$ Departamento de Ciencia de los Materiales, ETSI Caminos, Canales y Puertos, Universidad Politécnica de Madrid C/ Profesor \\ Aranguren s/n, 28040 Madrid, España
}

\begin{abstract}
The Hopkinson Bar has been widely used by many researchers for the analysis of dynamic properties of different brittle materials and, due to its great interest, for the study of concrete. In concrete structures subjected to high velocity impacts, initial compression pulses travel through the material leading to tensile stresses when they reach a free surface. These tensile efforts are the main cause of concrete fracture due to its low tensile strength compared to the compressive one. This is the reason why dynamic tests in concrete are becoming of great interest and are mostly focused in obtaining tensile fracture properties. Apart form the dynamic tensile strength, which has been widely studied by many authors in the last decades, the dynamic fracture energy presents an increased difficulty and so not too much experimental information can be found in literature. Moreover, up to date there is not a clear methodology proposed in order to obtain this parameter in an accurate way. In this work a new methodology for measuring the dynamic fracture energy is proposed by using the Hopkinson Bar technique. Initial tests for a conventional concrete have been carried out and the results for the dynamic fracture energy of concrete at different strain rates are presented.
\end{abstract}

\section{Introduction}

Nowadays concrete remains as the key material for building civil and military structures. Apart from the static characterisation, the analysis of dynamic properties of concrete is taking an important role. Possible explosions, accidents or terrorist threats demand an intense effort to protect civil and military structures. Achieving that, requires improving material models so that they can be capable of predicting structure's life, not only under static loads but also subjected to sudden dynamic impacts.

It has been demonstrated by many different authors that properties of concrete vary depending on whether a quasi-static or a dynamic analysis is performed [1]. In the case of the tensile strength, experimental tests performed have shown an important increase in this value, showing that more complex mechanisms take place under dynamic events, leading to a different behaviour and an important change in its properties. To begin with, loads are introduced in the structure in the form of short compression pulses which travel through the concrete material to be reflected at the free surfaces [2]. These compression pulses do not introduce a critical damage in the structure, instead, the reflected tensile waves constitute the initial step for its collapse. During the reflection process, compression pulses are transformed into tensile loads which will lead either to the spalling phenomenon causing the ejection of concrete debris or, eventually, to the formation of cracks and thus introducing an important non-visible damage across the whole structure [3].

\footnotetext{
*e-mail: v.rey@upm.es
}

Regarding the structural dynamic analysis, during the last decades high-strain-rate characterisation parameters, such as the dynamic tensile strength and fracture energy, have been widely studied. In the case of tensile strength, many authors have proposed different ways for measuring it [4][5][6][7][8]. An important amount of experimental data has been collected, showing in most of the cases a significant exponential growth of the corresponding values with increasing strain rate. By contrast, there is not to much experimental data for fracture energy or at least a clear methodology proposed for measuring this parameter in an accurate way. Even though energy experiments have already been conducted by some authors [1][8][9][10], a deepest study and more experimental data are needed in order to have a clear understanding of the energetic response of concrete material under dynamic loads.

In this work a new methodology is proposed for measuring dynamic fracture energy of concrete by using the Modified Hopkinson Bar technique together with notched specimens. Initial results obtained for a conventional concrete are also presented.

\section{Experimental methods}

\subsection{Concrete manufacturing and curing}

Dynamic tested specimens consist of concrete cylinders of $450 \mathrm{~mm}$ length and $69 \mathrm{~mm}$ in diameter. PVC tubes with this diameter were used to manufacture the specimens. Regarding the high length-to-diameter ratio, which can cause difficulties during vibration, a self-compacting concrete (SCC) was used in order to obtain an homogeneous mix inside the PVC mould. The dosage of this con- 
crete is given in Table 1 . The maximum aggregate size used in concrete was $12 \mathrm{~mm}$.

Table 1. Concrete dosage.

\begin{tabular}{lccc}
\hline Component & Volume $\left(\mathrm{m}^{3}\right)$ & Dosage $(\%)$ & Density $\left(\mathrm{kg} / \mathrm{m}^{3}\right)$ \\
\hline Cement & 114 & 11 & 360 \\
Crushed stone & 317 & 32 & 799 \\
Sand & 357 & 36 & 940 \\
Filler & 45 & 4 & 116 \\
Water & 147 & 15 & 147 \\
SP (ADVA) & 5 & 1 & 6 \\
Pores & 15 & 2 & 0 \\
Total & 1000 & 100 & 2367 \\
\hline
\end{tabular}

\subsection{Static tests}

Previous to dynamic Hopkinson Bar tests, compression, Brazilian and three-point-bending (TPB) tests were performed in order to have a quasi-static characterisation. Specimens devoted to static tests were directly obtained from the previous concrete cylinders, cut to the required length depending on the test carried. For compression and Brazilian tests, cylindrical specimens with a lengthto-diameter ratio of 2:1 were used.

The static configuration for a TPB test as well as the load-displacement curve obtained are shown in Figure 1. The total fracture energy $\left(E_{F}\right)$ can be obtained from these

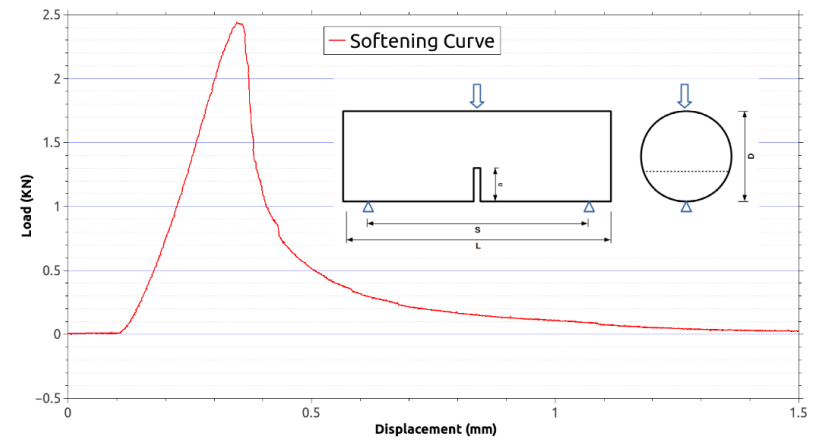

Fig. 1. Load-displacement curve for a three-point-bending test in cylindrical notched concrete specimen.

curves by numerical integration, computing the area under them. This area represents the consumed energy during the fracture process and, dividing by the fracture area $\left(A_{F}\right)$, the specific fracture energy is calculated $\left(G_{F}\right)$. In Table 2 results for both total and specific energiy are given for all the tests. The properties for the SCC used both for static and dynamic tests are shown in Table 3.

\subsection{Dynamic tests}

The experimental scheme used in this work is shown in Figure 2. This experimental device is composed of a steel conic projectile of $225 \mathrm{~mm}$ length inside the air cannon and a high-yield-stress steel incident bar of $1000 \mathrm{~mm}$
Table 2. Fracture energy results for quasi-static three-point-bending tests.

\begin{tabular}{ccccccccc}
\hline Test & $L(\mathrm{~mm})$ & $D(\mathrm{~mm})$ & $s(\mathrm{~mm})$ & $a(\mathrm{~mm})$ & $A\left(\mathrm{~mm}^{2}\right)$ & $A_{F}\left(\mathrm{~mm}^{2}\right)$ & $E_{F}(\mathrm{~J})$ & $G_{F}(\mathrm{kN} / \mathrm{mm})$ \\
\hline Test 1 & 345 & 69 & 276 & 15 & 3739.28 & 3139.60 & 0.638 & 0.000203 \\
Test 2 & 375 & 69 & 276 & 15 & 3739.28 & 3139.60 & 0.491 & 0.000156 \\
Test 3 & 390 & 69 & 276 & 15 & 3739.28 & 3139.60 & 0.587 & 0.000187 \\
\hline Mean & & & & & & & & 0.000182 \\
Stdev & & & & & & & & $2.4 \mathrm{E}-05$ \\
\hline
\end{tabular}

Table 3. Concrete properties. Characteristics of the self-compacting concrete

\begin{tabular}{lc}
\hline Constitutive equation & Linear Elastic \\
Maximum aggregate size $(\mathrm{mm})$ & 12 \\
Density $\left(\mathrm{Kg} / \mathrm{m}^{3}\right)$ & 2.202 \\
Wave speed (m/s) & 4081 \\
Dynamic Young Modulus (GPa) & 36.93 \\
Poisson's Ratio & 0.3 \\
Compressive Strength $(\mathrm{MPa})$ & 25.78 \\
Tensile Strength $(\mathrm{MPa})$ & 2.8 \\
Dynamic Tensile Strength $(\mathrm{MPa})$ & 12 \\
\hline
\end{tabular}

length and $22 \mathrm{~mm}$ diameter. The incident bar is instrumented with one strain gauge in the middle length. At the end of this bar, the concrete specimen is placed. The interaction between the incident bar and specimen is accomplished thanks to a metallic cylindrical piece embedded in one face of the concrete specimen. The concrete bar is also instrumented with a strain gauge at the central section. The testing device is completed with a Vision Research highspeed camera, model Phantom V12.1. The images from the high-speed camera are post-processed using Ncorr, a Digital Image Correlation (DIC) software [11]. The set-up at the laboratory can be seen in Figure 3.

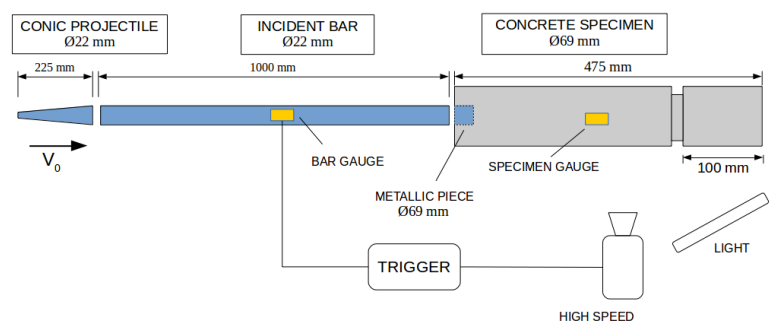

Fig. 2. Experimental scheme.

\subsection{Specimen's design}

Regarding the energy release, in concrete subjected to spallation, as the tensile pulse travels through the specimen after reflecting at the free end, energy relief is going to take place in two forms (Figure 4). First, in the form of complete surface cracks leading to spallation, and second, as micro-cracking along the length of the specimen and between surface cracks. If more than one fracture occurs during the process, energy is going to be dissipated not only in the fracture positions but also between these positions due to continuous micro-cracking. Accounting for all this surface micro-cracking is not feasible and so estimation of the fracture area will not be accurate enough to compute the specific fracture energy. 


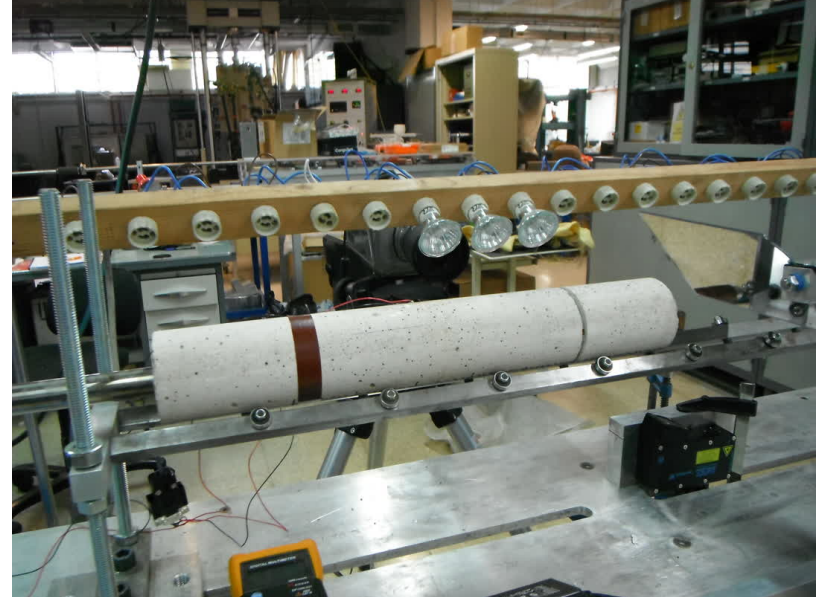

Fig. 3. Notched specimen, light and HS camera ready for testing.

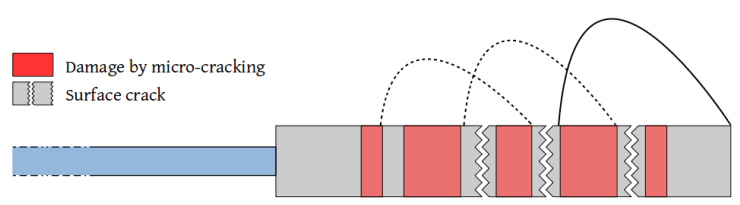

Fig. 4. Damage introduced in concrete due to tensile reflected pulses.

Reducing the previous inaccuracies, which could lead to wrong estimations of the fracture energy, is one of the main objectives of this work. It is known that, completely avoiding micro-cracking when subjecting concrete bars to tensile pulses is not possible but several actions can be adopted in order to minimize this last effect. First, the use of a conic projectile allows introducing triangular-shaped pulses into the specimen which ensure that, at every instant, only one section of the specimen will be subjected to the maximum peak, as opposite to rectangular-shaped pulses in which the stress is constant along the whole timepulse, meaning that at a every instant an entire portion of the specimen will be subjected to the maximum stress. The existence of a single maximum peak enables locating the stress and prevents other sections from damage. Moreover, when the incident wave is reflected, tensile stresses are going to arise progressively while returning back from the free end. This results in a critical fact as it enables to determine, previous to the test, the most suitable position for the notch. Second, notched specimens are used. The idea behind the notch is to be able to locate the fracture in just one section so that the fracture area is previously known with complete accuracy. Moreover, by introducing a notch with a reduced section, tensile stresses are locally increased at that point, overpassing in that particular section the dynamic tensile strength of concrete $\left(f_{c t d}\right)$ while in the rest of the specimen's sections, stresses are kept under its tensile strength, minimizing multi-fracture and the diffused fracture energy relief.

Several steps are needed to have an optimal specimen's design in order to accurately measure the dynamic fracture energy. The key starting point for these tests is knowing the dynamic tensile strength of the tested concrete. This parameter has been determined in previous spalling tests over the same type of SCC. These tests were carried out as part of a previous work [12]. Once dynamic tensile strength is known, either a numerical simulation or an analytical process can be performed to estimate the position of the initial in-time fracture. Finally, after locating the fracture position, specimens were finally designed and manufactured, placing the notch at $100 \mathrm{~mm}$ from the free end.

\subsection{Dynamic Fracture Energy}

The method proposed in this paper for the precise determination of the fracture energy is based on the analysis of the evolution of the energies inside the concrete specimen after the projectile's impact in one end. When the incident bar hits the concrete specimen, a compression pulse is transmitted. This pulse travels across the specimen and, thanks to its high compressive strength, concrete is able to withstand this pulse without damage. More over, for the analysis of the fracture energy, the maximum pulse peak introduced in concrete is kept below the tensile strength. The total energy introduced in the specimen is determined by that initial pulse. Any compression in the particles of the specimen will entail first, a movement in the particles leading to a kinetic energy (1) and second, a deformation of the infinitesimal sections, which results in an internal energy (2).

$$
\begin{gathered}
E_{k}=\frac{1}{2} \int v^{2} d m \\
E_{i}=\frac{1}{2} \int \sigma \cdot \varepsilon d V
\end{gathered}
$$

As the whole process takes place under elastic regime, kinetic and internal energies are equivalent and so both can be derived by just using the information of strains contained in the pulse. Given a constant section along the specimen and knowing that the length and time travelled by the pulse across the specimen are related by the wave speed, numerical integration of the records of one strain pulse can be done leading to

$$
E_{i}=\frac{1}{2} E \cdot A \cdot c \sum \varepsilon(t)^{2} \Delta t
$$

And so the total initial energy introduced into the specimen can be computed

$$
E_{T}=E_{k}+E_{i}=2 \cdot E_{i}=E \cdot A \cdot c \sum \varepsilon(t)^{2} \Delta t
$$

\subsection{Energy balance}

In the experimental tests carried out in this work, the projectile was shot with controlled air pressure in order to limit the maximum stress peak $\left(\sigma_{\text {peak }}\right)$ reached by the pulse. After hitting the concrete specimen, the compression pulse travels through it, reflects at the free end and returns back as a tensile pulse. Given that the tensile peak remains controlled below the dynamic tensile strength, no fracture is expected until reaching the notch. Due to the 
reduced area at that section, tensile stresses are increased and so a unique fracture takes place. The cohesive nature of the fracture process enables part of the returning tensile pulse passing through the cracking area to the next part of the specimen while part of the pulse remains trapped in the ejected free end. This means that part of the energy returns to the initial part of the specimen and part is retained in the ejected end (Figure 5). As it will be later demonstrated, the retained energy at the free end has no elastic or internal component,just kinetic. The velocity of the ejected end just after the fracture, and thus the kinetic energy $\left(E_{f e}\right)$, can be measured by using DIC over the images of the high-speed camera. The initial $\left(E_{0}\right)$ and residual $\left(E_{r}\right)$ energies can be computed thanks to the records at the strain gauge. These energies can be decomposed into kinetic and internal components. The procedure to derive these two energies is the same as the one explained in previous sections. Finally, a small portion of the energy $\left(E_{F}\right)$ is consumed to produce the fracture surface.

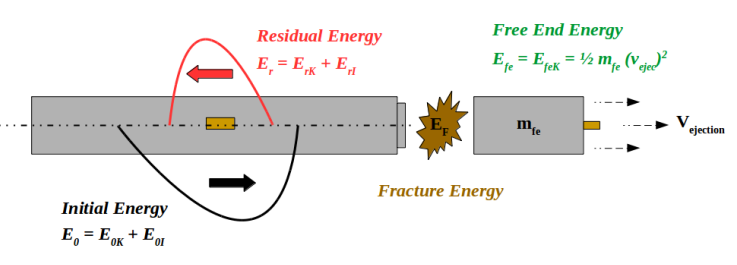

Fig. 5. Energy balance during fracture process.

The energy balance for the whole fracture process corresponds to

$$
E_{0}=E_{r}+E_{f e}+E_{F}
$$

From all of them, $E_{0}, E_{r}$ and $E_{f e}$ can be directly measured as explained, allowing $E_{F}$ to be derived:

$$
E_{F}=E_{0}-E_{f e}+E_{r}
$$

Finally, the specific fracture energy can be obtained dividing the total fracture energy by the fracture surface.

\section{Numerical simulations}

Numerical simulations were performed during this work for several reasons. All numerical simulations were carried using a special subroutine for the concrete material, programmed by D. Cendón and G. Morales [13] and implemented in the explicit finite element solver LS-DYNA. This constitutive model is based on the Cohesive Crack Model with Strong Discontinuity Approach developed by J. Planas and J.M. Sancho [14] and has been previously implemented for finite element analysis of concrete under quasi-static regime [15]. One of the possibilities of this model is that it can be used to perform numerical simulations of concrete elements subjected to explosions being, if desired, dependent on the strain rate. The model can be described as linear elastic with failure just under tensile efforts once the maximum principal stress is exceeded. To do so, different shapes of softening curves can be selected in order to fully represent the cohesive behaviour of the elements.

An initial model of the specimen without a notch was developed with the aim of locating the approximate position of the first crack caused by reflected tensile stresses. After that, a second model was developed for a notched specimen. The position of the notch was conditioned by the results of the previous simulation. The notch was placed at $100 \mathrm{~mm}$ from the free end.

The described energy balance in previous sections was also validated using numerical simulations. One of the hypothesis adopted in that balance was that the energy of the ejected free end after spallation corresponds only to kinetic energy, as no elastic wave was retained in this free end. This effect could be confirmed analysing the energies of the different parts of the numerical model. Figure 6 shows the evolution of the isolated energies corresponding to the free end. It can be seen that for this part of the model, the total energy corresponds entirely to kinetic energy.

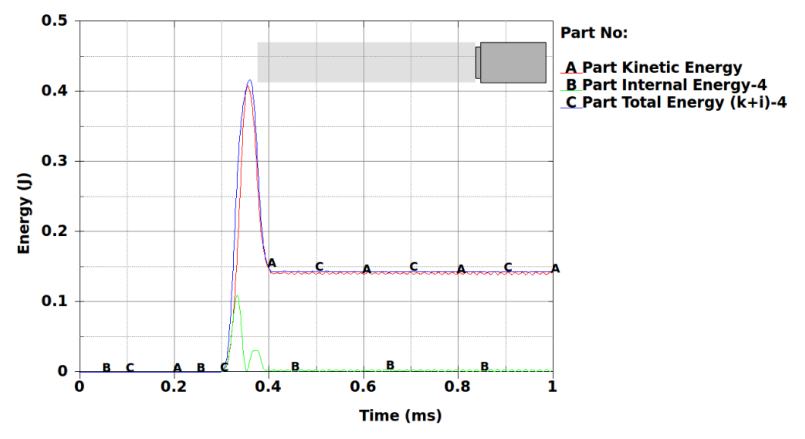

Fig. 6. Evolution of energies in the numerical model for the free end.

All the simulations were performed with the same pulse as the one introduced for the unnotched model and, as expected, the fracture was located at the notch, with no other fractures located at other sections. The procedure described for experimentally obtaining the dynamic fracture energy of concrete was tested using the results from the numerical simulations as if we did with the records in the gauges. The model was fed with a specific fracture energy set to $210^{-4} \mathrm{kN} / \mathrm{mm}$.

With the records from a finite element at the middle part of the specimen, initial and residual strains were plotted and, applying the methodology described, $G_{F}$ was derived, giving a value of $2.0110^{-4} \mathrm{kN} / \mathrm{mm}$, which coincides with the defined for the model, validating the proposed methodology.

These simulations were also useful to check the influence of the pulse magnitude introduced in the system over the specimen's damage (Figure 7) and consequently, over the estimated fracture energy. It is important to note that high-controlled pulses have to be used, always limiting the maximum compression peak below the dynamic tensile strength. Figure 7 shows that if non-controlled pulses are used and so the maximum tensile peak introduced in concrete is not kept below the dynamic tensile strength, several sections are going to be damaged without the for- 
Table 4. Energy estimations for different impact pulses.

\begin{tabular}{cccccccc}
\hline Model & Relative Impact Velocity & $E_{0}(\mathrm{~J})$ & $E_{r}(\mathrm{~J})$ & $E_{f e}(\mathrm{~J})$ & $E_{F}(\mathrm{~J})$ & $A_{F}\left(\mathrm{~mm}^{2}\right)$ & $G_{F}(\mathrm{kN} / \mathrm{mm})$ \\
\hline 1 & $1 \mathrm{~V}$ & 0.474 & 0.202 & 0.13 & 0.142 & 706.85 & $2.01 \mathrm{E}-04$ \\
2 & $1.5 \mathrm{~V}$ & 1.073 & 0.259 & 0.61 & 0.204 & 706.85 & $2.89 \mathrm{E}-04$ \\
3 & $2 \mathrm{~V}$ & 1.896 & 0.343 & 1.19 & 0.363 & 706.85 & $5.14 \mathrm{E}-04$ \\
4 & $2.5 \mathrm{~V}$ & 2.965 & 0.418 & 2.05 & 0.497 & 706.85 & $7.03 \mathrm{E}-04$ \\
\hline
\end{tabular}

mation of specific open fractures, impossible to account during a real experimental test and so leading to incorrect approaches of the fracture energy (Table 4) due to the wrong estimation of the fracture surface (Models 2, 3 and 4). Conditions of real experiments were adjusted in order to match the requirements accomplished in model 1 of the numerical simulations, where a fully controlled pulse is simulated.

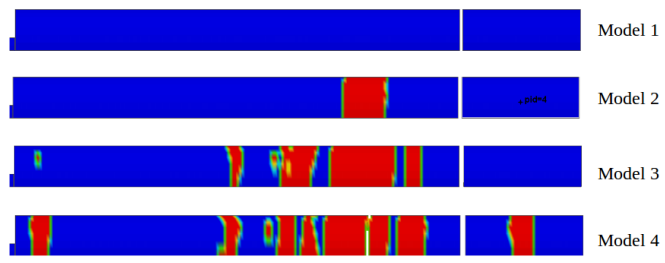

Fig. 7. Damage introduced in the model depending on the magnitude of the pulse used.

\section{Results}

A total of 10 initial tests were performed in the Modified Hopkinson Bar at varying strain rates. The aim of this preliminary tests is to give an initial idea of the accuracy of the methodology proposed.

Due to the complexity of the test not all of them were carried successfully. Unsuccessful tests showed either multiple or out-of-the-notch fractures, not allowing for an accurate estimation of the energy. Successful tests showed unique and clear fracture is located at the notch position without any other fractures. From the performed tests, five were carried correctly (A17, A18, B2, B5, B6), and using the information recorded at the strain gauges, $E_{0}$ and $E_{r}$ were integrated. The analysis of the images from the highspeed camera allows estimating the velocity of the ejected free end just after the fracture process, and knowing the mass of the ejected piece the kinetic energy is easily obtained.

In Table 5 the strain rate for every performed test is shown as well as the measured energies and the estimated fracture energy. The estimation of the strain rate was done using DIC, analysing the strains with time at the notched section. DIC was also used to control that no fracture occurs in more than one section.

In Figure 8 results for the all the energies involved in the process as a function of the strain rate are plotted. An exponential trend can be identified for every energy, increasing as the strain rate grows.

In Figure 9 an identical plot is shown for the specific fracture energy. These values have been computed from the previous fracture energy dividing by the considered
Table 5. Energy results for the performed tests.

\begin{tabular}{ccccccc}
\hline Test & $E_{0}(\mathrm{~J})$ & $E_{r}(\mathrm{~J})$ & $E_{k}(\mathrm{~J})$ & $E_{F}(\mathrm{~J})$ & $G_{F}(\mathrm{kN} / \mathrm{mm})$ & Strain rate $\left(s^{-1}\right)$ \\
\hline A16 & 1.246 & 0.16 & 0.866 & 0.22 & $7.79 \mathrm{E}-05$ & 150 \\
A17 & 1.057 & 0.24 & 0.227 & 0.59 & $2.09 \mathrm{E}-04$ & 17 \\
A18 & 1.079 & 0.159 & 0.288 & 0.631 & $2.23 \mathrm{E}-04$ & 50 \\
B1 & - & - & - & - & - & - \\
B2 & 1.765 & 0.384 & 1.114 & 0.266 & $9.41 \mathrm{E}-05$ & 80 \\
B3 & - & - & - & - & - & - \\
B4 & - & - & - & - & - & - \\
B5 & 1.575 & 0.492 & 0.717 & 0.365 & $1.29 \mathrm{E}-04$ & 75 \\
B6 & 3.197 & 0.72 & 1.309 & 1.167 & $4.13 \mathrm{E}-04$ & 110 \\
\hline
\end{tabular}

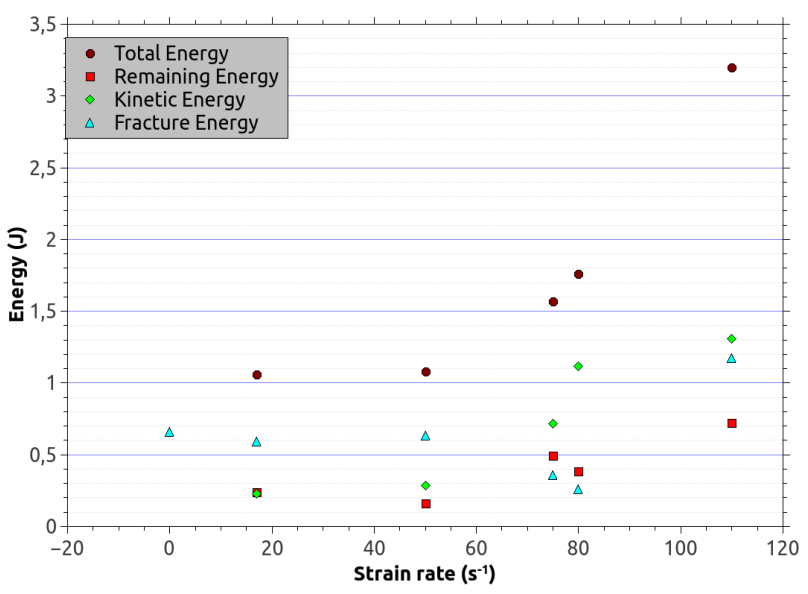

Fig. 8. Energies involved in the fracture process.

fracture surface, which in all the cases was given by the area of the notch $\left(3739 \mathrm{~mm}^{2}\right)$. In this same plot the value of the fracture energy obtained from quasi-static threepoint-bending tests is included as a threshold dashed line $\left(210^{-4} \mathrm{~J} / \mathrm{mm}^{2}\right)$. Regarding the specific fracture energy, a critical strain rate can be identified for a value of $100 \mathrm{~s}^{-1}$. This value can be seen as the transition from quasi-static to dynamic behaviour. In this sense, the initial quasi-static regime, ranging from static tests to strain rates up to $80 \mathrm{~s}^{-1}$, is characterised for keeping the fracture energy around the referred static value, while the dynamic behaviour can be identified in strain rates over $100 \mathrm{~s}^{-1}$, with a significant increase in the estimated fracture energy. Even though there are just some values of the fracture energy, these can be taken as an initial approximation for the behaviour of concrete, helping in the identification of the transition from static to dynamic regime.

\section{Conclusions}

The methodology proposed allows measuring the Dynamic Fracture Energy of concrete using an indirect approach based in an energy balance. A modified Hopkinson Bar scheme has been used to develop tensile stresses in concrete and, using a conical projectile, triangular pulses were introduced into the specimen. With the help of numerical simulations, an optimal notched specimen design was reached. This, combined with the local stress increment at that section, enables the use of tensile pulses with a maximum stress peak below the dynamic tensile strength 


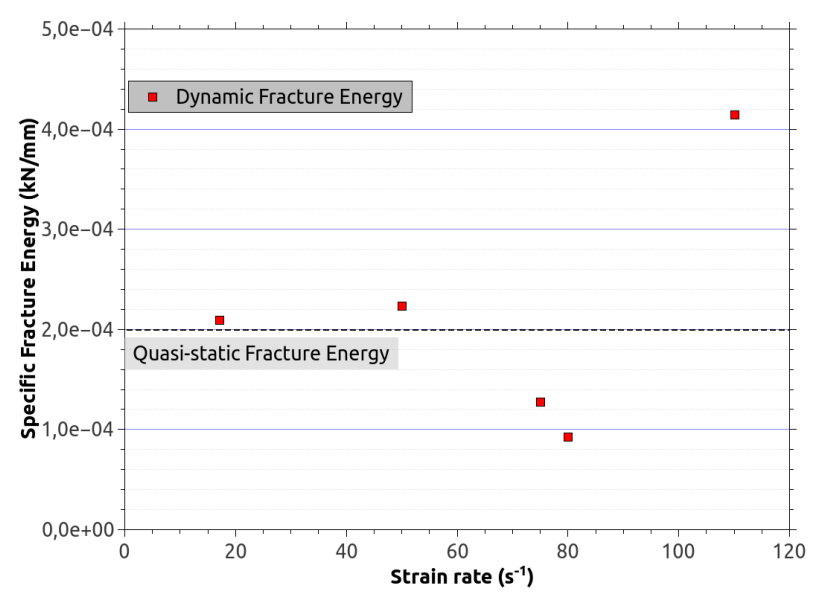

Fig. 9. Results for the Specific Fracture Energy of concrete.

of concrete, preventing the rest of the sections from damaging.

Numerical simulations were fundamental in order to validate the methodology proposed. They also showed how uncontrolled pulses can lead to wrong estimations of the fracture energy, caused mainly by multi-fracture events.

After validating the methodology, a set of 10 experimental tests were carried out. In five of them a unique fracture at the position of the notch was achieved. From the experimental results, two regimes were identified:

- A quasi-static regime, defined by a strain rate under 80 $\mathrm{s}^{-1}$, where values of $\mathrm{G}_{F}$ are in the order of the values obtained from TPB tests.

- A dynamic regime, defined by a strain rate above $80 \mathrm{~s}^{-1}$, where values of $\mathrm{G}_{F}$ are increased with respect to quasistatic values

The analysis of all of the energies also showed a raising trend with the strain rate. Compared to the static values from TPB tests, a DIF of 2 has been found for a conventional concrete. This factor was reached for a strain rate close to $120 \mathrm{~s}^{-1}$.

These preliminary tests were carried to validate the methodology proposed, and so an additional complete experimental campaign should be done to verify the results obtained. An increase in the strain rate reached would also be desirable in order to have a complete dynamic characterisation of concrete under a wide variety of strain rates.

The authors would like to acknowledge the financial support obtained from the spanish "Ministerio de Economía y Competitividad" through the national project BIA2014-54916-R.

\section{Author contribution statement}

F. Gálvez is responsible for the conception and design of the experimental device; V. Rey de Pedraza performed the experimental work and the analysis and interpretation of data; D. Cendón was responsible for the numerical simulations and worked in the analysis and interpretation of data; Both F. Gálvez. and D. Cendón. drafted the article and revisited it critically.

\section{References}

1. H. Schuler, C. Mayrhofer, K. Thoma, International Journal of Impact Engineering 32, 1635 (2006)

2. J.A. Zukas, High velocity impact dynamics (Wiley, New York, 1990), ISBN 978-0-471-51444-2, oCLC: 21445603

3. D.O. Dusenberry, Handbook for Blast Resistant Design of Buildings (John Wiley \& Sons, 2010), ISBN 978-0-470-17054-0, google-Books-ID: 19Gr55RPcRQC

4. F. Gálvez Diaz-Rubio, J. Rodriguez Pérez, V. Sánchez Gálvez, International Journal of Impact Engineering 27, 161 (2002)

5. J.R. Klepaczko, A. Brara, International Journal of Impact Engineering 25, 387 (2001)

6. B. Erzar, P. Forquin, Experimental Mechanics 50, 941 (2010)

7. H. Wu, Q. Zhang, F. Huang, Q. Jin, International Journal of Impact Engineering 32, 605 (2005)

8. J. Van Doormaal, J. Weerheijm, L. Sluys, Journal de Physique IV Colloque 04, C8 (1994)

9. A. Brara, J.R. Klepaczko, International Journal of Impact Engineering 34, 424 (2007)

10. J. Weerheijm, J.C.A.M. Van Doormaal, International Journal of Impact Engineering 34, 609 (2007)

11. J. Blaber, B. Adair, A. Antoniou, Experimental Mechanics 55 (2015)

12. V. Rey-De-Pedraza, D. Cendón, V. Sánchez-Gálvez, F. Gálvez, Philosophical Transactions of The Royal Society A Mathematical Physical and Engineering Sciences 375, 20160174 (2017)

13. G. Morales Alonso, phd, E.T.S.I. Caminos, Canales y Puertos (UPM) (2013), http : //oa .upm . es/16028/

14. J.M. Sancho, J. Planas, A.M. Fathy, J.C. Gálvez, D.A. Cendón, International Journal for Numerical and Analytical Methods in Geomechanics 31, 173 (2007)

15. J.M. Sancho, J. Planas, D.A. Cendón, E. Reyes, J.C. Gálvez, Engineering Fracture Mechanics 74, 75 (2007) 